TRANS · núm. $24 \cdot 2020$

NOTAS · 469-481
Este trabajo explora el rol que en la poesía mapuche contemporánea tiene la práctica de la traducción colaborativa, entendida como uno de los actuales esfuerzos de los intelectuales, escritores y traductores del pueblo-nación mapuche por revitalizar su lengua vernácula, el mapuzungun, en el ámbito de la cultura y la literatura. Examinaremos el caso de la antología de poesía mapuche escrita por mujeres, Kümedungun/ Kümewirin (2010) a cargo de la poeta y lingüista Jaqueline Caniguan y un equipo de colaboradores. La recreación de la lengua a través de neologismos, el posicionamiento ideológico de los traductores y las reflexiones plasmadas en los paratextos de la antología serán analizados en torno de un ejemplo específico: la traducción colaborativa al mapuzungun del poema «Mi destino» de Adriana Paredes Pinda.

PALABRAS CLAVE: traducción colaborativa, poesía mapuche, translaboration, revitalización lingüística.

\title{
Traducción colaborativa en la poesía mapuche: un aporte a la revitalización del mapuzungun
}

\author{
Melisa Stocco \\ CELLPA-Universidad Nacional de la \\ Patagonia San Juan Bosco/CONICET,
} Argentina

\section{Collaborative Translation in Mapuche Poetry: a contribution to the revitalization of Mapuzungun}

In contemporary Mapuche poetry, the practice of collaborative translation holds a special place in that it can be understood as one of the current efforts of intellectuals, writers and translators of the Mapuche nation to revitalize their vernacular language, Mapuzungun, within the cultural and literary fields. We will examine the case of the anthology of Mapuche poetry written by women, Kümedungun / Kümewirin (2010), where most Mapuzungun versions were translated by the poet and linguist Jaqueline Caniguan and her team of collaborators. The recreation of the language through neologisms, the ideological positioning of the translators and the reflections on the process developed in the paratexts of the anthology will be analyzed around a specific example: the collaborative translation of the poem «Mi destino» by Adriana Paredes Pinda.

KEY WORDS: collaborative translation, Mapuche poetry, translaboration, linguistic revitalization. 


\section{LA TRADUCCIÓN COLABORATIVA COMO MULTIPLICIDAD DE PRÁCTICAS}

La revisión de todas las premisas básicas de la traducción para lograr una mayor comprensión de su diversidad de formas y prácticas a lo largo del tiempo incluye repensar, según María Tymoczko, la idea de que traducir es una labor individual (2005: 1088). La imagen del traductor solitario se contrapone a una realidad en la que, tanto a nivel literario como pragmático, tal trabajo requiere de diversos colaboradores (Cordingley y Frigau-Manning, 2017: 1). La historia europea medieval y renacentista, por ejemplo, prueba que la labor traductora compartida era la regla más que la excepción. Es el caso de lo que Belén Bistué ha estudiado en el contexto del Renacimiento europeo como «equipos de traductores» que trabajaban en versiones multilingües, con bagajes culturales distintos y conocimientos de lenguas diversas (2013:1).

En este sentido es que los estudios de traducción han comenzado a prestar atención al fenómeno de la llamada traducción colaborativa: un campo abierto y dinámico de prácticas de traducción que componen un trabajo colectivo. Entre esta miríada de formas colaborativas, se encuentra la traducción orientada al aprendizaje de lenguas asistido por computador (CALL), la traducción en línea mediante colaboración abierta distribuida (crowdsourced), la traducción en comunidad, principalmente la realizada por amateurs en el contexto interactivo de la Web 2.0., entre otras. La inclusión de la traducción colaborativa en estudios de procesos más amplios ha llevado incluso a la reciente elaboración del concepto de «translaboration», entendido como un «tercer espacio» que permite explorar y articular conexiones, comparaciones y zonas de contacto entre la traducción y la colaboración (Alfer, 2017: 286). Cabe mencionar también en esta línea de estudios la noción de «multiple translatorship» de Hanne Jansen y Anne Wegener (2013: 5) como modo de caracterizar la naturaleza frecuentemente colaborativa de la traducción, la cual no deja de lado las posibles situaciones de conflicto y división de este trabajo conjunto.

Dentro de las categorías de análisis que Jansen y Wegener delimitan dentro de la traducción con múltiples traductores; a saber, el proceso, el producto y la autoridad, esta última es analizada a partir de una adaptación de la tipología de autorías de Harold Love: precursora, ejecutiva, declarativa y revisora (23). Las estudiosas retoman esta clasificación para analizar las diversas agencias implicadas en la tarea traductora y reconocen que la interacción fundamental se produce entre los traductores ejecutivo, es decir, quien efectivamente realiza el trabajo lingüístico; declarativo, quien aparece en la portada como persona a cargo de la traducción; y revisor; es decir, quien realiza una labor editora (Jansen y Wegener, 2013: 24).

Más allá de las nuevas formas de traducción surgidas del ámbito digital, la colaboración en la traducción literaria también sigue siendo un campo en continuo desarrollo. Dentro de este contexto, la traducción colaborativa de poesía ha recibido, puntualmente, mucha menor atención, ya que, tal como señalan Steven Cranfield y Claudio Tedesco en coincidencia con la hipótesis de Lawrence Venuti, el estatus de la poesía como capital económico es muy minoritario (2017: 305). ¿Qué formas translaborativas adopta la traducción de poesía en equipo en tanto tarea inserta en un contexto sociohistórico, editorial y lingüístico específico, y qué implicancias éticas, lingüísticas y culturales conlleva? Estas preguntas, llevadas al ámbito de la poesía mapuche contemporánea, suponen atender, además de las especificidades culturales y procesuales interlingüísticas, el rol ocupado por la traducción colaborativa en el entramado de resistencias 
políticas y lingüísticas del pueblo mapuche y su potencial transformativo en la recuperación y revitalización de una lengua subalternizada como el mapuzungun. Por lo tanto, este trabajo se propone la combinación metodológica del close reading, de corte comparativo o «estereoscópico» en términos de Marilyn Gaddis Rose (1997: 90), que se centra en las tensiones, divergencias y complementariedades semánticas entre textualidades en traducción, con el distant reading, que toma en consideración sus condiciones sociohistóricas de producción y se deriva principalmente del análisis paratextual presentado a continuación.

\section{KÜMEDUNGUN/KÜMEWIRIN (2010), UNA EXPERIENCIA DE TRADUCCIÓN COLABORATIVA EN LA POESÍA MAPUCHE}

Las expresiones que la traducción toma en la poesía mapuche contemporánea, la cual cuenta con un vasto corpus bilingüe mapuzungun-español, van desde la más común de la autotraducción, hasta experiencias de retraducción y traducción colaborativa. Las múltiples antologías de poesía mapuche, así como diversos poemarios individuales, dan cuenta de esta diversidad de prácticas en las que se combinan la traducción del propio autor con distintas modalidades de la traducción alógrafa, a cargo de expertos en mapuzungun asignados a tal fin. En lo que concierne a la traducción colaborativa, la práctica tiene como referente a Jaqueline Caniguan (Puerto Saavedra, 1974): poeta, lingüista, docente y activista de la revitalización del mapuzungun en Gulu Mapu, actual territorio chileno.

Caniguan ha conjugado su actividad poética con estudios lingüísticos tanto en Chile, donde se formó como Profesora de Castellano en Temuco, como en México, donde se graduó como
Magíster en Lingüística Indoamericana. Su formación y labor académica se encuentran íntimamente ligadas a la militancia que Caniguan lleva adelante desde hace varios años como educadora y activista en organizaciones sociales mapuche-lafkenche para promover la supervivencia del mapuzungun en las comunidades, puesto que, como ella misma señala: «Esta lengua se encuentra minorizada y en situación de desmedro frente al castellano» (2007, en línea).

Como traductora, Caniguan fue encargada de verter al mapuzungun los poemas de autoras mapuche de la antología Kümedungun/Kümewirin (2010), curada por Fernanda Moraga y Maribel Mora Curriao. Este volumen cuenta con tres apartados: el primero está dedicado a un conjunto de $\ddot{u} l$ o cantos de mujeres recopilados durante los siglos XIX y XX; el segundo compendia la obra de poetas contemporáneas pioneras que editan sus trabajos durante las décadas de los setenta y ochenta —entre ellas, Sonia Caicheo, Jeannette Hueitra, Rayen Kvyeh, María Teresa Panchillo-; y el tercero y último destaca a un grupo de poetas mapuche actuales, publicadas desde la década del noventa hasta la actualidad del libro, es decir, fines de la primera década de los dos mil: Liliana Ancalao, Viviana Ayilef, Graciela Huinao, Roxana Miranda Rupailaf, Ivonne Coñuevar, Faumelisa Manquepillán, Adriana Paredes Pinda, entre otras.

En nuestro análisis de la traducción colaborativa que tiene lugar en esta obra, prestaremos especial atención a los elementos paratextuales, en tanto para los estudios de traducción históricos y descriptivos tienen un papel central en la contextualización del proceso de traducción y las normas y convenciones que subyacen la producción del texto meta (Toledano Buendía, 2013: 150). El título, subtítulos, notas aclaratorias, notas de traductor, prefacios, entre otros, cons- 
472 tituyen un conjunto de dispositivos literarios que, además de consumar la recepción del texto como libro (Genette, 2001 [1987]: 7), indican la importancia no solo del producto de la traducción sino también de su acto, lo que, de acuerdo con Jansen y Wegener, permite reconocer los diferentes intereses y formas de trabajo involucrados en la traducción (2013: 7).

Como se indica desde la portada del libro, $K \ddot{u}$ medungun/Kümewirin cuenta con la «versión mapuzungun» de todos los textos, tanto los ül tradicionales como los poemas contemporáneos, a cargo de Jaqueline Caniguan, salvo cuando se indique lo contrario en nota a pie de página. Sin embargo, en el paratexto titulado «Para leer los poemas en mapuzungun y la historia de su traducción» (Caniguan en Mora Curriao y Moraga, 2010: 21-25), la propia Caniguan aclara que el trabajo realizado fue concretado «en conjunto con otros lamgen» (21), es decir, con otros «hermanos y hermanas mapuche», ya que lamgen es un término utilizado en mapuzungun para referirse a otros pares étnicos. Esta es la primera mención al carácter colaborativo de la traducción de la antología. Observamos que, en términos de Jansen y Wegener, la traductora declarativa es Caniguan, aunque, las tareas de traductores ejecutivos - $-\mathrm{y}$ probablemente revisores - también involucran a otros actores. Más adelante, Caniguan da los nombres de dichos participantes:

Lo vi como una oportunidad e invité a mi gran amiga Catalina Marileo, de Pu Budi, a que iniciáramos este recorrido. Durante muchas tardes de verano, acompañadas del mate, conversábamos cada poema, buscábamos palabras y creábamos otras. Luego se nos sumaron los jóvenes pewenche Felidor Manquepi y Juan Manquepi, quienes nos regalaron su chedungun, variante del mapuchedungun. Y así fuimos traduciendo a nuestro idioma los poemas de las escritoras mapuches (Caniguan en Mora Curriao y Moraga, 2010: 21-22).
Aquí Caniguan no solo menciona a sus colaboradores, sino que también describe las tareas que conformaron el proceso. La conversación, el diálogo ameno alrededor del mate que se comparte como se comparte la lectura de los cantos y poemas, se cifra como método de creación de neologismos o de búsqueda de las mejores palabras para la traducción al mapuzungun -lo que puede haber involucrado, además, el uso de obras de referencia. La traductora también aclara que la contribución esencial de Felidor y Juan Manquepi fue la de sumar la perspectiva de la variedad de la lengua mapuche conocida como chedungun, con lo cual la colaboración aquí podría orientarse al aporte de mayor diversidad dialectal a las traducciones, que volverían más abarcativo su público de destino. Caniguan refiere algunas de las dificultades y de los hallazgos de este proceso, por ejemplo, el neologismo que crearon para designar el género literario poético en mapuzungun: «Al principio no sabíamos cómo decir «poesía», porque comprendíamos que un poema escrito no es un $\ddot{u l}$. [...] Decidimos entonces decir kümewirin «el escrito hermoso»y así comenzamos a llamarle» (22).

Por otro lado, Caniguan reconoce que las traducciones fueron creciendo en dificultad a medida que se acercaban cronológicamente al presente. Es decir, los ül tradicionales, recopilados en castellano, eran reconocidos con facilidad por los intérpretes y asociados a recuerdos de infancia, con lo cual el trabajo fue una suerte de traslado que prácticamente prescindió de la búsqueda de palabras: «rápidamente podíamos llevarlo[s]1 a nuestra lengua» (22). En contraposición, los poemas contemporáneos requirieron de más reflexión y de «detener la tarea para comprender» (22). La principal dificultad fue de tipo léxica: «hubo muchas palabras del español

I El resaltado es nuestro. 
que no conocíamos» (22). Esta situación llevó a la búsqueda en diccionarios y a la posterior discusión del término más acorde en mapuzungun, el cual, de no ser hallado, suponía la elaboración de un neologismo o, en su defecto, como veremos más adelante, de su directa omisión.

La construcción de nuevas palabras en mapuzungun supone un punto de polémica a resaltar. La formulación en lengua mapuche de ideas y conceptos creados originalmente por las poetas en castellano implica un posicionamiento de los traductores en torno de ideas como la legibilidad del texto en mapuzungun y los grados de «extranjerización» de la palabra mapuche o de «domesticación» de los conceptos provenientes del español, todo lo cual se relaciona con el fin último de revitalización lingüística del mapuzungun. En este sentido, Caniguan y su equipo optaron por la innovación basada en la idea de la vitalidad de la lengua mapuche. La lingüista destaca que algunas de las traducciones «pueden incluso resultar incomprensibles» para «el más purista de los hablantes» mapuche (23), sin embargo, la creación de nuevas construcciones y palabras responde a la capacidad de adecuación de una lengua viva, como el mapuzungun, a los tiempos que corren. Caniguan recuerda cómo, en los comienzos del contacto entre mapuche y españoles, la lengua incorporó préstamos léxicos tales como sumpiru («sombrero»), waka («vaca»), kawellu («caballo»), los cuales no fueron en desmedro de su fuerza expresiva, sino que, por el contrario, la enriquecieron (23).

El proceso de creación de neologismos supuso, a criterio de Caniguan, un esfuerzo por «no $m a$ puchizar conceptos». Esta orientación a la innovación léxica parece no conllevar para la traductora una mayor «castellanización» de la lengua, sino una apuesta por la capacidad de creación y adaptación del mapuzungun que lo actualice y revitalice. En esta línea, las motivaciones de la traducción colaborativa se enlazan con lo que Elisa Loncón y Francesco Chiodi plantean como la necesidad de renovar y actualizar los recursos lexicales del mapuzungun a fin de darle expresión a las transformaciones y adaptaciones de la sociedad mapuche y trascender las restricciones provocadas por la estigmatización y acoso sufrido por una lengua restringida a ciertos usos específicos (1999: 6).

A lo largo del texto, Caniguan resalta una y otra vez la principal motivación para llevar a cabo estas traducciones: «el deseo de que el $m a$ puchedungun siga presente» (22), «nuestro afán primero es la vida del idioma mapuche» (23). La operación de la traducción se erige, entonces, como herramienta de revitalización lingüística de un mapuzungun «que como sabemos se encuentra cada vez menos en la voz de nuestros niños y parece retraerse en la voz de los ancianos» (21). Las traducciones son «un aporte a la vida de la lengua», y una «invitación al reencuentro y a la readquisición de la lengua» (25).

A continuación, analizaremos el trabajo realizado sobre un poema de Adriana Paredes Pinda. En él observaremos de qué manera la traducción colaborativa de Jaqueline Caniguan, Catalina Marileo, Juan Manquepi y Felidor Manquepi se desarrolla en línea con los desafíos marcados por la propia lingüista en el paratexto ya citado. Es decir, examinaremos los casos de neologismos y la manera en que amplían o restringen los sentidos originales del texto en castellano a la vez que modifican y enriquecen el acervo léxico de la lengua mapuche. A su vez, nos detendremos a analizar las omisiones operadas en la traducción a fin de comprender los desafíos y limitaciones que la intraducibilidad supone para el equipo traductor. 


\section{TRADUCCIÓN COLABORATIVA DE UN POE- MA DE ADRIANA PAREDES PINDA: NEOLO- GISMOS Y OMISIONES}

La antología Kümezungun/Kümewirin incluye varios poemas de Adriana Paredes Pinda, entre ellos el titulado «Mi destino» que constituye el germen de una sección del poemario Parias zugun (2014), publicado cuatro años más tarde por la autora ${ }^{2}$. La traducción de Caniguan y su equipo se titula «Tañi mongen» literalmente traducible como «Mi vida» (Paredes Pinda en Mora Curriao y Moraga, 2010: 422-427).

A continuación, desglosaremos el análisis comparativo del texto original en castellano y su tra- ducción al mapuzungun en secciones y acompañaremos la versión mapuche con una traducción de estudio propia para una comprensión más cabal de lo vertido por los traductores.

«Por el pálpito de un beso» se omite, aunque no queda claro por la presencia de la palabra «kiñe» en el siguiente verso si esto es deliberado o se trata de una errata de edición. A su vez se decide utilizar el verbo nepen, «despertar», en voz activa («nepeleyngün») para traducir la construcción pasiva «levantados por», lo que lleva a considerar la posibilidad de que los traductores hayan estimado de especial potencia la imagen de los ancestros ya difuntos - el morfema -yem asílo indica-despertando al amanecer, en con-

\section{Tañi Mongen}

Ngülfual tañi piwke ñi kuku ka ñi

chuchu engu

ta kurükaniru ñi mollfüng mew

Takulen ta ran mew

Müllmüllmeken madkin mew ka

pu liwen

Pu fütakecheyem nepeleyngün

Kiñe ${ }^{3}$

\section{Traducción de estudio}

Untaré el corazón de mi abuela paterna y de mi abuela materna

con la sangre de carnero negro

Me visto con plata

Tiritando en la niebla y el amanecer

los ancestros despiertan

[Uno]

\section{Mi destino}

Untar el corazón de mis abuelas

con sangre de negros carneros

prenderme

de plata

titilando entre niebla y aura

los fvtakechem

levantados por el pálpito de un beso.
2 El poema en cuestión forma parte, con modificaciones y agregados, de la secuencia que va desde las páginas $88 \mathrm{a}$ 101 del libro Parias zugun (2014).

3 En este verso parecen faltar palabras que corresponderían a la traducción del verso par «por el pálpito de un beso». Posible errata en la edición. sonancia con la fuerza de la acciones poéticas de carácter ritual que evoca toda la estrofa.

En este pasaje los traductores no omiten ningún elemento ni crean neologismos para la versión en lengua mapuche, sin embargo, rea- 
Wirarü tañi üy,

Rangipuntu-pingün

Fey mew müleyu inchiw

Ngülfukunuy

Petu ñi lanun

\section{Traducción de estudio}

Grita mi nombre, en medio de la noche/ a medianoche, dijeron

entonces nos quedamos los dos

Se ungió

antes que me muera
Grita mi nombre

en lo alto de la noche- dijeron

y me quedaré contigo

aunque

muerte

sea ungida lizan unos cambios temporales en los verbos «me quedaré» y «sea ungida» que unifican en la forma básica traducible por defecto como pretérito perfecto simple al castellano («muleyu» y «ngülfukunuy»). En el caso de la frase «me quedaré contigo», además del cambio de futuro a pretérito, se muestra una modificación de la persona verbal, de primera a dual (muleyu iñchiw). En cuanto al presente del subjuntivo «sea ungida», en voz pasiva en la versión castellana, los traductores optan por la forma verbal básica en voz activa del mismo verbo con el resultativo -künu utilizado en la estrofa anterior al referirse a los corazones untados con sangre: ngülfun, «untar, ungir». Esto marca otra forma de unificación semántica de la traducción, aunque la versión original de Paredes Pinda refiera a verbos diferentes.
Las decisiones creativas de los cotraductores van al encuentro de la característica complejidad de la poética de Paredes Pinda que muchas veces dificulta la búsqueda de términos equivalentes en mapuzungun. Es probable que, por este mismo motivo, toda una sección del poema no fuera traducida al mapuzungun, a saber los versos: «que expiara todas mis transgresiones/y amansara el lomo de las azuladas madres. / La lengua rosada pulpa de ignominia y tempestades/ no prescribe». Las traducciones muestran divergencias no solo léxicas, sino también a nivel sintagmático, al modificar la estructura versal para traducir un núcleo de sentido que se expresa de manera muy diferente al original. Es así como en los versos «Sin embargo, nací/ con las yemas/ balbuceantes/ sin la moneda de la sangre pura»,
Müna weda kay llegpan

Nielan remapuche mollfüng

$\tilde{N} i$ kalül mew

\section{Traducción de estudio}

y muy mal nací

no tengo sangre mapuche pura en mi cuerpo
Sin embargo, nací

con las yemas

balbuceantes

sin la moneda de la sangre pura

que expiara todas mis transgresiones

y amansara el lomo de las azuladas madres.

La lengua rosada pulpa de ignominia y tempestades no prescribe 
476 los traductores se resuelven por «Müna weda kay llegpan/Nielan remapuche mollfüng/ Ni kalül mew» que literalmente sería traducible como «y muy mal nací/ no tengo sangre mapuche pura/ en mi cuerpo». La versión mapuche resigna la traducción de «yemas balbuceantes», o la referencia a «la moneda de la sangre pura», conjunto de términos que configuran en el original una imagen táctil que representa la búsqueda frustrada de una identidad basada en un supuesto ideal de pureza, para construir una estructura más directa en la contundencia del mensaje.
Paredes Pinda, quien ha definido su identidad «champurriada» como el «ser hija de dos lenguas y el habitar/ser habitada por dos o más corazones», entendiendo a esta duplicidad «como [dos] formas de pensamiento, de sentir y comprender la vida» (Paredes Pinda 2013: 15). En la traducción de los versos «Qué somos/ sino/ parias/villanos/ desgarrados/ sin el tatuaje de la eternidad», los términos omitidos son «villanos» y «desgarrados». En su lugar la versión en mapuche traduce «parias» como wariache, es decir, «gente de la ciudad», lo cual revela una
Müna weda femniengen pu pajina mew, Rewe, ¿Chem am iñchiñ?

Nienolu chew ñi mülen

Wariache, ngelay kimün,

Ngelay aflayay wiritukuy trawa mew

Allküñmalaenew ñi ñuke,

Allküñmalaenew lüfal kiñe pimün metawe

\section{Traducción de estudio}

Muy mal estoy así entre las páginas

Rewe, ¿quiénes somos?

Sin tener donde estar

gente de ciudad, sin conocimiento

sin el eterno dibujar en la piel

[No hay, no concluye, (se) dibuja en la piel]

No me escucha mi madre

No me escucha encender un soplo metawe he sido condenada a desgarrarme, rewe entre las páginas

Qué somos

sino

parias

villanos

desgarrados

sin el tatuaje de la eternidad.

Que no me sienta madre

que no me encienda

el soplo

del metawe
En los siguientes versos los cotraductores modifican el orden sintagmático $\mathrm{y}$, en consecuencia, la literalidad del texto meta. Respecto de lo primero, «rewe entre las páginas» ve alterado su orden: el rewe, centro ceremonial de las prácticas rituales mapuche, pasa a ser, por la disposición versal, un vocativo, al que se le hace la pregunta existencial por el ser («Rewe, ¿Chem am iñchiñ?), temática de enorme centralidad en la poesía de interpretación específica de lo que implica ser «paria» para los traductores: vivir en contextos urbanos alejados del territorio ancestral como resultado de las diásporas forzadas del pueblo mapuche. Asimismo, este término es combinado con la frase «ngelay kimün», traducible como «sin sabiduría», aquella que se desarrollaría en contacto con las prácticas culturales del pueblo mapuche en un contexto comunitario y, una vez 
Wariache ñi dungun,

Müpafküley ka ünatun

Rütreenew pu lifüro, ta ñi ñamal ñi üy

\section{Traducción de estudio}

La palabra de la gente de ciudad

aletea/aleteó y muerdo/mordí

los libros me empujan para que pierda mi nombre que muda

mi lengua paria en aleteo y mordisco

me puja los códices que desdicen mi nombre más, en el territorio. En cuanto a la forma de traducir «sin el tatuaje de la eternidad», los traductores eligen combinar varios verbos, entre ellos, aflayay, forma negativa del verbo afn, «concluir» (Augusta 1991:3), lo que podría entenderse como «sin fin», para traducir «eternidad», y wiritukuy, «dibujar» (130) combinado con trawa mew, «en la piel», para referirse al «tatuaje».

Nuevamente la modificación sintagmática conforma una imagen divergente en el texto meta. En lugar de «mi lengua paria», los cotraductores eligen retomar la idea de wariache para pensar la alusión que «paria» hace al «sin territorio» en el mundo mapuche y su ligazón a la diáspora urbana. Así, el término wariache conjugado al posesivo $\tilde{n} i$ que puede ser tanto de primera como de tercera persona, con dungun, «lengua o palabra», se abre a una idea más amplia de pertenencia de esa lengua champurriada a toda la gente de la ciudad, no solo al yo poético que figura en el original. Asimismo «en aleteo y mordisco» es reemplazado por dos verbos que pueden ser traducidos en forma pretérita o presente, uno en tercera persona (müpafküley) y otro en primera (ünatun) donde el yo vuelve a tomar presencia. Por otra parte, el último verso también evidencia una decisión de corte ideológico en la traducción al elegir el verbo ñamün,
Ah müna weda kewün, nienoli.

Pimunge, pimunge,

Chumgechi kam puruleymi wiñotual tami reñma,

Namikay awkiñ, pültrelewey wilufforo,

tañi yomkuku Filipa,

\section{Traducción de estudio}

Ah, muy mala lengua, si no te tuviera

Sopla, sopla

Como danzaste/danzas para recuperar

tu familia

El eco desaparece, se quedó colgado el diente brillante/

hueso resplandeciente,

mi tatarabuela paterna Filipa
Ah lengua mísera de mí

si no te tuviera

sopla

sopla

que has de danzar tu recuperada

casta

de soterrados ecos

pendiéndose

en los molares

florecientes

de Filipa, mi tatarabuela 
478 «perder» para traducir «desdecir»: la cultura letrada de los libros en lengua castellana produce la pérdida del propio nombre ancestral, una ambigüedad que la poesía de Paredes Pinda complejiza insistentemente.

Además de nuevos cambios como la incorporación del interrogativo «chumngechi kam»y el cambio temporal de la forma básica «puruleymi» (danzaste/ danzas) para traducir la perífrasis verbal con valor de futuridad «has de danzar», el neologismo reconocible en este segmento es «wilufforo», la forma elegida para traducir la frase «molares florecientes». Foro puede significar tanto «hueso» como «diente». El término ülnga, además, puede equivaler a «muela» (Augusta 1991: 274), lo cual haría referencia más directa a los «molares». Sin embargo, los traductores eligen la palabra foro y la combinan con wiluf que significa «brillante, resplandeciente» (261). De esta manera, los traductores eligen una forma entendible como «huesos resplandecientes» para referir a los «molares» de los que habla el texto original.

Otros dos casos de neologismo que van asociados en el poema son los vocablos creados para traducir el verso «por porfiada y lujuriosa».
El primer adjetivo se traduce como maykenulu, el cual se compone del verbo mayn, «obedecer», el habituativo - $k e$, la forma negativa - nu y el sufijo - lu de participio, lo cual podría traducirse como «la que no obedece». Por otra parte, «lujuriosa» se conforma con el verbo fütangen, «tener marido, estar casada» (48) y el sufijo agentivo - $f e$ que designa personas con habilidad en cierto trabajo o que tienen la costumbre de realizar una determinada acción (Augusta 1903: 247). La palabra resultante, fütangefe, puede interpretarse como «que suele tener o buscar marido», como una manera de referir al deseo sexual propio del término «lujuriosa» en castellano. En ambos casos la estrategia de acuñación de neologismos concuerda con la derivación de lexicalización espontánea (Loncón y Chiodi 1999: 109).

La creatividad en la traducción de la última estrofa se revela en la composición del primer verso al aunar la imagen poética del original, «te recuperaré y mi lazo rodeará ...», con el verbo defmutungelu encabezado por la raíz def, «soga». También es destacable la elección de los términos «tami mongen karü mawida dungun» para referirse al «aura» de la lengua mapuche, cuyas «sílabas» Paredes Pinda compara con «magno-
Tañi kewün ta tripayay ka ngillayal wekufe mew, Tañi müleal kewün, wilufke ünüm ñi neyen,

Katrikatrituymaenmew tañi ñmal, Katrikatrilungey, pingün.

Maykenulu ka fütangefe wewtuayu,

\section{Traducción de estudio}

Mi lengua se saldrá para pactar con el wekufe El vivir de la lengua, el aliento de pájaros relámpago

Me cortan intensamente para que pierda, es cortada, dicen

La que no obedece y suele buscar marido que mi lengua ha de salirse de sí misma

y pactar con el wekvfe que la ampara su reverberación de pájaros y aromas que mi lengua ha de partirse —ha de partirse — dicen por porfiada y lujuriosa 
Defmutungelu tami mongen karü mawida dungun.

Ah, tañi Pinda kewün, tañi kewün ka kom pu domo

Nienulu kuñiltukun

\section{Traducción de estudio}

enlazaré tu vida

lengua de la montaña/ del bosque verde

Ah, mi Pinda lengua, mi lengua [y de]

todas las mujeres

sin compasión te recuperaré

y mi lazo

rodeará acaso el aura de tus sílabas magnolias

ah mi pinda lengua

lengua mía y de todas sin misericordia lias». Hay aquí una decisión creativa por parte de los traductores de asociar la idea de «aura» con «mongen», «vida», y la imagen vegetal y de enraizamiento a la tierra que la poeta confiere a la lengua, con los términos «karü mawida», «montaña/bosque verde» - la propia ambivalencia del término mapuche enriquece aún más el efecto poético-, lo que genera una metáfora diversa pero complementaria a la original.

\section{CONCLUSIONES}

En general, el trabajo de traducción observado en este poema muestra dos procedimientos destacables: el de los neologismos que se concentran en la segunda parte del texto, y el distanciamiento prácticamente total de la equivalencia semántica y léxica en ciertos pasajes. Estas instancias de la traducción evidencian alteraciones sintagmáticas y omisiones de algunos tramos de los versos originales, posiblemente ligados a la determinación de secciones intraducibles en la lengua meta por parte de los traductores.

El trabajo colaborativo con la traducción de un poema de la complejidad de «Mi destino» de Adriana Paredes Pinda, evidencia la creatividad del proceso en la medida en que las conversaciones y búsquedas conjuntas del equipo de traductores deriva en la acuñación de nuevas palabras en mapuzungun para suplir los casos de intraducibilidad que se presentan. Sin embargo, como también demuestra la lectura estereoscópica (Gaddis Rose 1997: 90) del poema, se dan casos en los que se decide omitir partes del texto original en la versión en mapuzungun por no hallar posibilidades de paridad semántica y léxica con aquél. El trabajo conjunto de Caniguan con su grupo de traductores también resulta en decisiones como la de traducir «paria» como wariache, que muestran un importante distanciamiento de los conceptos del original y revelan un posicionamiento ideológico probablemente negociado entre los intérpretes del texto, que supone una concepción de la vida urbana como desarraigada y excluida del territorio ancestral como reaseguro de conexión con el mundo mapuche.

El anterior análisis evidencia que la traducción colaborativa no es solo relevante en cuanto a los diversos roles que pueden haber tenido los distintos traductores - algo sobre lo que solo podemos especular, más allá de los datos provistos por la nota aclaratoria escrita por Caniguan-, 
480 sino que también demuestran, como plantean Cordingley y Frigau Manning, la importancia de pensar la traducción desde una perspectiva que supere la epistemología del individuo y ofrezca una mirada compleja de la imbricación y fusión que subyacen y producen el trabajo colectivo (2017: 24).

Además, las motivaciones sociales, políticas y culturales de proyectos como el realizado por Caniguan y su equipo son tan importantes como el producto mismo del trabajo para comprender el alcance de la traducción colaborativa en contextos como el de una literatura minoritaria. El deseo de contribuir a la revitalización del mapuzungun ha movido a este grupo de personas a trabajar en equipo para crear versiones de poemas de autoras mapuche en la lengua ancestral. Dicho esfuerzo pretende acoplarse al de los posibles lectores que se acerquen a los textos, a fin de aportar un corpus de lectura a su proceso de (re) adquisición del mapuzungun. En este sentido, la traducción colaborativa de la poesía mapuche contemporánea puede entenderse como una acción que busca extender su carácter colaborativo a los lectores mismos, a quienes se invita a superar las mismas dificultades de comprensión y encarar su acercamiento a los textos con el mismo afecto por la palabra revitalizada.

\section{BIBLIOGRAFÍA}

Alfer, Alexa (2017): «Entering the Translab: Translation as Collaboration, Collaboration as Translation, and the Third Space of 'Translaboration'», Translation and Translanguaging in Multilingual Contexts 3/3, 275-290.

Augusta, Fray Félix José de (1903): Gramática Araucana, Valdivia: Imprenta Central, 1903.

(1991): Diccionario Araucano. Mapuche-Español.
Español-Mapuche, Temuco: Editorial Kushe.

BistuÉ, Belen. (2013): Collaborative Translation and Multi-Version Texts in Early Modern Europe, Londres y Nueva York: Routledge.

Caniguan, Jaqueline (2010): «Para leer los poemas en mapuzungun y la historia de su traducción», Mora Curriao, Maribel y Fernanda Moraga (Eds.), Kümedungun/Kümewirin. Antología poética de mujeres mapuche (siglos XIX y XX), trad. mapuzungun: Jaqueline Caniguan, Santiago de Chile: LOM, 21-25. (2007): «Un gran aporte en mi trabajo con el mapudungun», Garabide, <www.garabide.eus/ blogak/bidaidetzak/2007/11/27/jaqueline-caniguan-un-gran-aporte-en- mi- trabajo-de-revitalizacion-del-idioma-mapuche>

Cordingley , Anthony y Céline Frigau Manning (2017): Collaborative Translation: From the Renaissance to the Digital Age, London: Bloomsbury.

Cranfield, Steven y Claudio Tedesco (2017): «Reformulating the Problem of Translatability: A Case of Literary Translaboration with the Poetry of Francisco Brines». Translation and Translanguaging in Multicultural Contexts 3 (3), 304-322

GADDIS, Rose Marilyn (1997): Translation and Literary Criticism, Manchester: St. Jerome.

Genette, Gerard (2001 [1987]): Umbrales, México y Buenos Aires: Siglo Veintiuno.

Jansen, Hanne y Anne Wegener (2013): Authorial and Editorial Voices in Translation 1: Collaborative Relationships between Authors, Translators and Performers, Montréal: Éditions québécoises de l'oeuvre.

Loncón, Elisa y Francesco Chiodi (1999): Crear nuevas palabras. Innovación y expansión de los recursos lexicales de la lengua mapuche. Temuco: UFRO/CONADI.

Paredes Pinda, Adriana (2010): «Mi destino» en Maribel Mora Curriao y Fernanda Moraga (ed.), Kümedungun/Kümewirin. Antología poética de mujeres mapuche (siglos XIX y XX), trad. mapuzungun: Jaqueline Caniguan, Santiago de Chile: LOM, 422427.

(2013): Epu rume zugu rakiduam: Desgarro y florecimiento. La poesía mapuche entre lenguas. Tesis de 
Doctorado, Valdivia: Universidad Austral de Chile. (2014): Parias zugun, Santiago de Chile: LOM.

Toledano Buendía, Carmen (2013): «Listening to the voice of the translator: A description of translator's notes as paratextual elements», Translation \& Interpreting 5 (2), 149-162.

түмосzко, María (2005): «Trajectories of Research in Translation Studies», Meta: Translator's Journal 50/4, 1082-1097. 